Meta

Journal des traducteurs

Translators' Journal

\title{
Formation et pratique de la traduction professionnelle en Belgique francophone
}

\section{Louis Yvon Chaballe et Jean Klein}

Volume 39, numéro 1, mars 1994

La traduction et l'interprétation dans la Belgique multilingue

URI : https://id.erudit.org/iderudit/002211ar

DOI : https://doi.org/10.7202/002211ar

Aller au sommaire du numéro

Éditeur(s)

Les Presses de l'Université de Montréal

ISSN

0026-0452 (imprimé)

1492-1421 (numérique)

Découvrir la revue

Citer cet article

Chaballe, L. Y. \& Klein, J. (1994). Formation et pratique de la traduction professionnelle en Belgique francophone. Meta, 39(1), 69-77.

https://doi.org/10.7202/002211ar d'utilisation que vous pouvez consulter en ligne. 


\title{
FORMATION ET PRATIQUE DE LA TRADUCTION PROFESSIONNELLE EN BELGIQUE FRANCOPHONE
}

\author{
LOUIS YVON CHABALLE ET JEAN KLEIN \\ Chaballe Traductions \& Communications et École d' interprètes \\ internationaux de l'Université de Mons-Hainaut, Mons, Belgique
}

\section{INTRODUCTION}

Pays de dix millions d'habitants, la Belgique compte trois langues officielles: par ordre d'importance, le néerlandais, langue de la «Vlaamse Gemeenschap», le français, langue de la «Communauté française de Belgique» et l'allemand, langue de la «Deutschsprachige Gemeinschaft». L'autonomie linguistique et culturelle de ces trois communautés est inscrite dans la constitution belge et, en conséquence, ces communautés organisent toute la vie publique dans leur langue respective.

Par ailleurs, la Belgique, située au cœur de l'Europe, est le siège officiel et officieux de nombreuses organisations internationales: Communautés européennes, OTAN, Conseil de coopération douanière, Eurocontrol, ainsi que de centaines d'organisations satellites qui gravitent autour des grandes et pour lesquelles il est important d'être présent à Bruxelles.

Ceci explique le rôle essentiel que jouent les langues en général et la traduction en particulier dans la vie politique, administrative, économique, sociale, culturelle et quotidienne du pays, ainsi que le nombre de traducteurs et interprètes qui y exercent leur métier.

Exposer en quelques pages la formation et la pratique de la traduction professionnelle en Belgique francophone nous oblige à nous concentrer sur l'essentiel et à faire l'impasse sur de nombreux détails. Si nous avons effectué ce choix au risque d'être critiqué pour manque de complétude, c'est qu'il nous a semblé opportun de fournir au lecteur un tableau synoptique en la matière.

Notre propos s'articulera en deux volets : le marché de la traduction, d'une part, et la formation, d'autre part, cette dernière étant elle-même subdivisée en «formation ancienne» et «formation nouvelle».

\section{LE MARCHÉ DE LA TRADUCTION EN BELGIQUE FRANCOPHONE}

La décision d'analyser en premier le marché de la traduction en Belgique francophone n'est pas fortuite, car ce marché conditionne - qu'on le veuille ou non - la formation dispensée dans les instituts et écoles supérieures de traduction et d'interprétation. Certes, il ne s'agit pas de calquer servilement les programmes sur les besoins souvent mouvants du marché. Les grandes écoles doivent conserver leur liberté et la marge de manœuvre académique qui leur est propre. Elles ne peuvent donc se transformer en sous-traitants du marché. Par ailleurs, cependant, elles ne peuvent ignorer les lois de ce marché au risque de s'enfermer dans une tour d'ivoire et de sombrer dans un académisme fatal. Elles devront donc trouver une adéquation aussi parfaite que possible entre les exigences d'une formation universitaire et celles de l'exercice d'une profession intellectuelle, dont le degré de complexité et de technicité reste très largement méconnu du public, y compris des donneurs d'ordres. 


\section{Le marché des organisations internationales}

Elles emploient essentiellement des traducteurs fonctionnaires qui traduisent vers leur langue maternelle. L'objectif est d'assurer à la fois la rentabilité et la qualité. Ces postes sont très recherchés, car, outre la stabilité d'emploi, les traitements sont quatre à cinq fois supérieurs à ceux des fonctionnaires nationaux. Mais il y a peu d'élus, car la politique actuelle vise à ne pas créer des services dont la taille rendrait la gestion difficile, voire impossible. Le nombre de fonctionnaires, recrutés par concours, est donc volontairement limité, ce qui oblige les Communautés européennes à confier à la sous-traitance les traductions que leurs services ne peuvent assurer.

En outre, dans certains cas, le recrutement de fonctionnaires est soumis à un contingentement national. Ceci concerne les pays qui ont une même langue officielle, comme la France et la Belgique, par exemple. En l'occurrence, il est évidemment exclu que des Belges francophones trustent tous les postes de la division française.

Côté sous-traitance, les Communautés européennes sont en train de réorganiser le secteur. Elles ont lancé un appel à manifestation d'intérêt qui sera suivi d'un appel d'offres permettant de constituer une réserve de traducteurs indépendants ou de bureaux de traduction auxquels les Communautés confieront certaines de leurs traductions. À titre informatif, signalons que quelque 3000 candidats ont répondu à cet appel : 1460 traducteurs individuels et 321 bureaux de traduction ont été retenus durant la première phase. Actuellement les Communautés sous-traitent à l'extérieur environ $10 \%$ de leur volume. Il est question de porter ce chiffre à $20 \%$, soit un volume de travail non négligeable pour certains traducteurs francophones belges ${ }^{1}$.

Les langues sources sont essentiellement les huit langues des Communautés, le français étant la langue cible. En pratique, la majorité des textes confiés aux traducteurs francophones belges sont des originaux anglais et allemands et, moins souvent néerlandais, espagnols, italiens, danois, portugais et grecs non seulement parce que ces originaux sont moins fréquents, mais aussi parce que les règles de la Commission des Communautés européennes imposent, pour adopter un texte au niveau des commissaires, que celui-ci soit disponible en anglais, allemand et français. Il importe aussi de considérer que l'élargissement de la CEE à des pays nordiques ou à des États d'Europe orientale multipliera certes les langues usitées dans le grand marché, mais conduira, selon les dires des responsables communautaires, inéluctablement, à une réduction des langues de travail. Le français, l'anglais et l'allemand ont un avenir assuré. Notons enfin que les derniers concours de recrutement de traducteurs imposaient aux candidats francophones de posséder l'anglais et/ou l'allemand dans leur combinaison linguistique.

\section{Le marché belge}

Il faut distinguer le marché intrabelge et le marché international, c'est-à-dire celui des pays et partenaires étrangers avec lesquels traitent les Belges ou les étrangers (État, entreprises, personnes privées) installés en Belgique.

Examinons d'abord le marché intrabelge. Contrairement à ce qui se passe dans un pays unilingue, la fonction publique belge est un gros employeur de traducteurs. Tout texte officiel de portée nationale - on devrait peut-être dire fédérale pour respecter la nouvelle réalité politique de la Belgique - doit être obligatoirement traduit dans l'autre grande langue du pays, le français ou le néerlandais, et souvent dans la troisième langue nationale, l'allemand.

En outre, les relations entre communautés (flamande, francophone et germanophone) et régions linguistiques (française, néerlandaise, bilingue de Bruxelles-Capitale, allemande) imposent très souvent la traduction de textes de toutes sortes. Dans la région de BruxellesCapitale, par exemple, tous les écrits et documents officiels doivent être disponibles en français et en néerlandais. 
Cela suppose un nombre élevé de traducteurs qui, non seulement traduisent de la langue source vers leur langue maternelle, mais aussi inversement quand cela est nécessaire. Tous ces traducteurs ne sont pas des diplômés de l'enseignement supérieur. C'est pourquoi il existe en la matière trois catégories de fonctionnaires : les traducteurs, titulaires d'un diplôme de l'enseignement secondaire ou d'un graduat $(\mathrm{bac}+2)$, les traducteurs réviseurs, titulaires du titre de licencié $(b a c+4)$ et les traducteurs directeurs. Il est donc normal d'être engagé dans la fonction publique, à la sortie des études, comme traducteur réviseur, ce qui en soi est une aberration, car, par définition, un traducteur réviseur est un traducteur chevronné, capable de juger et d'améliorer, si besoin est, les traductions réalisées par d'autres. Mais, sur le plan du lexique, le petit royaume fédéral n'en est pas à une confusion près.

Le marché intrabelge privé est lui aussi florissant. Entreprises et personnes privées font régulièrement appel aux traducteurs, que ce soit pour la commercialisation des produits dans une autre communauté linguistique, la publicité, l'établissement de contrats, le judiciaire (civil, pénal, commercial), les actes sous seing privé..., toute la vie intercommunautaire impose de recourir à des traducteurs.

De ce point de vue, la Belgique apparaît donc comme un des paradis translationnels de la planète. Il faut toutefois modérer cet enthousiasme, car le pays est petit et le marché n'est pas extensible au delà de certaines limites. De plus, pour les francophones belges, les langues pratiquées sont exclusivement le néerlandais et l'anglais, et, loin derrière, l'allemand. Cette réalité rend très délicat le choix des langues étudiées, et ce, pour plusieurs raisons. L'étude du néerlandais et de l'allemand est difficile pour un francophone. C'est une des combinaisons linguistiques où l'on enregistre le plus haut taux d'échecs. Par ailleurs, le néerlandais est une langue de petite diffusion, indispensable en Belgique et aux PaysBas mais parfaitement inutile ailleurs. Enfin, pour des motifs qu'il ne nous appartient pas de développer ici, le néerlandais est souvent une langue mal aimée.

Sur le marché international, le traducteur francophone belge doit surmonter un handicap initial : son existence est très souvent ignorée à l'étranger pour la simple raison qu'une personne ou une institution étrangère ayant besoin d'une traduction vers le français s'adressera à un traducteur ou à un bureau de traduction français. Quoi de plus naturel! Le traducteur francophone belge doit donc avant tout se faire connaître sur les marchés étrangers et y conquérir des clients fidèles. Ceci est possible par le biais des entreprises multinationales installées en Belgique ou par la réputation de qualité qu'a acquise le traducteur ou le bureau de traduction. À ce propos, la Belgique francophone peut se prévaloir de quelques belles réussites.

Quant aux types de textes à traduire, aux langues de travail les plus utilisées et aux postes de travail des traducteurs, une enquête sur le traducteur en Belgique réalisée en 1990 par Sabine Gérin nous révèle ce qui suit:

Classement des types de traduction selon la part qu'ils représentent dans le volume de travail total :

1) Juridique, administrative

2) Scientifique Chimie, physique Médecine, biologie

3) Technique $22,1 \%$ Technique générale

$11,3 \%$ Informatique, électronique

$6,4 \%$

4) Économique, commercial

5) Publicité, annonces

6) Catalogues, brochures

7) Correspondance 
8) Films, émissions TV, radio

9) Manuels didactiques

10) Discours

11) Bandes dessinées, livres pour enfants

12) Littéraire

Divers
$3,7 \%$
$1,6 \%$
$1,1 \%$
$1,0 \%$
$0,1 \%$
$1,6 \% 2$

Sur le marché belge de la traduction, la demande est, tout comme l'offre, très diversifiée. La part du lion revient toutefois aux textes spécialisés techniques, scientifiques, économiques et juridiques. Le marché est en effet de plus en plus spécialisé.

Les langues de travail les plus utilisées sont le français, le néerlandais, l'anglais et l'allemand, les deux premières étant «incontournables». Si la plupart des répondants traduisent vers leur langue maternelle, beaucoup travaillent vers une langue étrangère, d'où l'importance qu'il convient d'accorder à la connaissance active de la langue concerné $e^{3}$.

Le temps de la gomme et du crayon semble à jamais révolu. L'ordinateur personnel n'est plus un luxe, mais un outil indispensable au traducteur professionnel. L'informatique se répand jusqu'aux services publics. Les logiciels de traitement de texte les plus utilisés sont Wordperfect, Msword et Wordstar (sic).

La majorité des répondants remettent leurs traductions sur support électronique, le principal format utilisé étant le MS DOS.

Les communications connaissent également divers bouleversements, dont l'avènement du FAX n'est pas le moindre.

La publication assistée est moins utilisée. Cet investissement n'est en effet qu'à la portée des bureaux d'une certaine envergure.

La traduction automatique, quant à elle, ne semble pas remporter beaucoup de succès auprès des répondants. La machine, aussi perfectionnée soit-elle, est encore loin de pouvoir remplacer l'homme ${ }^{4}$ ?

Les résultats de cette étude belge dont sont tirés les extraits ci-dessus corroborent très largement ceux révélés par une étude allemande similaire publiée en résumé dans le «Mitteilungsblatt für Dolmetscher und Übersetzer $(M D \ddot{U}) »$ du Bundesverband der Dolmetscher und Übersetzer e.V. $(B D \ddot{U})$, journal de l'Association allemande des interprètes et traducteurs sous la plume de P. A. Schmitt (février 1990).

On peut donc en conclure, sans risque de se tromper lourdement, que la situation est similaire dans l'ensemble des pays de l'Europe occidentale, avec évidemment le nécessaire correctif des langues de travail les plus utilisées.

\section{La disproportion entre le nombre de diplômés et la demande du marché}

La partie francophone de la Belgique compte quatre instituts supérieurs de traducteurs et interprètes qui, bon an mal an, lancent sur le marché trois à quatre cents jeunes diplômés. C'est trop, beaucoup trop, le marché ne peut les absorber tous. Et pourtant, les chiffres officiels du Forem (agence pour l'emploi) ne font état que de 50 traducteurs demandeurs d'emploi (octobre 1992), ce qui, comparé à d'autres diplômés de l'enseignement supérieur, est extrêmement peu. Cela signifie-t-il que les quelque 300 autres travaillent comme traducteur? Loin s'en faut. Seule une très petite minorité embrasse la carrière. La plupart exercent leur art dans d'autres professions: professeur de langues vivantes, secrétaire (de direction), délégué commercial, membre d'un service de relations publiques, etc. D'autres poursuivent leurs études dans une autre filière, notamment les sciences économiques, politiques, administratives... Bref, tout semble bon pour ne pas se lancer dans le métier pour lequel ils ont été spécifiquement formés. Ceci s'explique en partie parce que les langues choisies, voire imposées, pendant les études ne répondent pas aux besoins du marché et parce que les étudiants avaient une idée fausse du métier de traducteur, mais débouche par ailleurs sur le paradoxe suivant: en dépit du grand nombre 
de diplômés, il y a, pour certaines langues, pénurie de bons traducteurs. C'est notamment le cas pour l'allemand.

\section{Les prix pratiqués}

Comme le titre et la profession de traducteur ne sont pas protégés en Belgique, la fourchette des prix pratiqués est très large et varie, en gros, entre 9 et $60 \mathrm{BEF}$ la ligne de 60 frappes et espaces selon les langues, la difficulté du texte, le service demandé (délai, mise en pages, relecture des épreuves) et la position du traducteur (fonctionnaire qui fait des traductions en plus, à titre privé, traducteur indépendant, bureau de traduction...). Certes, la «Chambre belge des traducteurs, interprètes et philologues», amalgame anachronique qui entrave toute (auto)régulation de la profession, publie des tarifs purement indicatifs qui ne reflètent pas la réalité des prix du marché. Enfin, la multiplication des apprentis traducteurs favorisée par la pléthore de diplômés suscite l'émergence d'un marché gris qui casse les prix, dégrade la qualité et discrédite l'image de la profession. La tactique du rabais financier et qualitatif appliquée par les jeunes traducteurs ou les traducteurs pas ou mal formés ne produit toutefois que des résultats éphémères. À la longue, seuls les bons traducteurs survivront. Les clients sont parfois conciliants, mais pas dupes.

\section{L'évolution du marché}

L'augmentation du volume des textes à traduire serait de l'ordre de $5 \%$ par an, mais ce n'est là qu'une estimation, car il est difficile de se faire une idée précise en la matière. L'internationalisation des marchés, la directive européenne obligeant à fournir une documentation dans la ou les langues du pays importateur - directive qui, par ailleurs, n'est toujours pas entièrement respectée - la législation sur la responsabilité du fait des produits défectueux contribuent au développement du marché de la traduction. Celui-ci reste toutefois lié aux fluctuations de la conjoncture. À l'heure actuelle, la récession et le marasme économique qui sévissent à l'échelle européenne et mondiale ne sont évidemment pas favorables aux secteurs des services en général et de la traduction en particulier.

\section{LA FORMATION}

Après avoir examiné le marché de la traduction en Belgique, venons-en à la formation et tentons d'en dégager les grandes lignes: quelle est-elle? Quelles sont ses lacunes? Dans quelle mesure répond-elle aux exigences du marché? Qu'est-on en droit d'en attendre?

\section{La formation ancienne}

L'objectif principal déclaré était d'apprendre à traduire et, pour ce faire, de se perfectionner aussi en langues maternelle et étrangères. L'acquisition d'un savoir et d'un savoirfaire se faisait par une formation adéquate portant sur:

\section{- la langue maternelle;}

- deux langues étrangères avec la possibilité d'une troisième en option ;

- des cours de matière (droit, économie, sociologie, linguistique, histoire des civilisations...);

- des cours de traduction vers la langue maternelle et vers deux langues étrangères ;

- l'acquisition d'une polyvalence et d'une culture générale de niveau universitaire.

Le but était de former des traducteurs généralistes, capables toutefois de traduire correctement des textes spécialisés dans les principaux domaines de l'activité humaine où le recours à la traduction est fréquent. Cet apprentissage s'effectuait généralement sur le tas, au hasard des textes choisis en appliquant le principe «c'est en traduisant qu'on devient traducteur». Ce principe a été respecté pendant des années et a d'ailleurs fourni 
d'excellents résultats chez les étudiants doués. II fonctionnait d'autant mieux que le nombre d'étudiants par classe était réduit, voire très réduit, et que l'enseignement, dispensé sous forme de séminaire ou d'atelier de traduction, était véritablement interactif. Hélas, ces temps sont révolus et, économies obligent, le nombre d'étudiants dans les classes de traduction pour les langues de grande diffusion atteint régulièrement les 30 unités et parfois plus. Dans ce cas, l'apprentissage sur le tas est devenu beaucoup plus aléatoire et peut dévier vers un élitisme de fait, car seuls les meilleurs sont capables de trouver leurs repères, d'abstraire, de généraliser, de transposer et d'appliquer à d'autres textes le savoir et le savoir-faire acquis au cours des textes précédents.

Il a donc fallu repenser la formation pour des raisons pédagogiques, d'une part, mais aussi pour répondre, d'autre part, aux exigences accrues du marché de la traduction.

\section{La formation nouvelle}

Elle ne supprime rien de la formation ancienne mais la renforce sur le plan de la recherche, de la rigueur, de la polyvalence et la complète en y intégrant l'aspect professionnel du «traduit». Elle comprend les éléments suivants :

\section{Réflexions sur l'opération traduisante}

Cette réflexion a lieu à partir de la littérature spécialisée. Il ne s'agit cependant pas ici de se plonger dans la lecture des théoriciens de la traduction dont les écrits, qui sont souvent d'un haut niveau scientifique, ont peu d'utilité pour le traducteur praticien. On serait presque tenté de leur suggérer d'abandonner le projet d'écrire un nouvel ouvrage théorique pour se consacrer à la traduction de quelques milliers de pages afin qu'ils appréhendent enfin la réalité de l'opération traduisante.

Heureusement, de bons praticiens devenus pédagogues ont rédigé d'excellents livres pratiques, tels les deux ouvrages de Daniel Gouadec (1989 et 1990) et celui de Christine Durieux (1988), par exemple, pour ne citer que trois ouvrages francophones parmi les plus récents. Ces ouvrages de base peuvent être complétés par une série d'articles traitant de langues, de domaines, de textes particuliers (Klein 1987) ou encore d'aspects spécifiques de l'opération traduisante. Il en existe. L'ensemble constitue une solide base de réflexion méthodologique et pratique et permet d'acquérir des «réflexes de traducteur» plus rapidement que par le simple apprentissage sur le tas, qui d'ailleurs les exercera et les renforcera.

\section{Méthodes et étapes de la traduction de textes en langue de spécialité}

Avant d'aborder la traduction proprement dite, il est nécessaire de maîtriser un certain nombre de prérequis. En fait, il s'agit d'apprendre la rigueur sur le plan de la réception (texte original) et de la production (texte traduit). Cette rigueur comprend la présentation, l'orthographe, la syntaxe, le style, le respect de la cohérence et de la logique... bref, c'est l'acquisition d'un savoir-être préalable à celle d'un savoir et d'un savoir-faire translationnel.

La pédagogie de ces prérequis repose sur trois principes. En premier lieu, le principe de faisabilité. Il est inutile de placer les étudiants devant des problèmes insolubles, par exemple en leur demandant de comprendre des textes sans leur donner les outils et les informations indispensables ou, dans un deuxième temps, en leur demandant de répertorier les ouvrages de référence dont ils auront besoin pour pouvoir comprendre correctement le texte. Ensuite, le principe errare humanum est. Tout apprentissage implique forcément le droit à l'erreur et il serait à la fois frustrant et contre-productif de sanctionner d'emblée les fautes commises. Elles doivent être signalées, expliquées et corrigées afin d'être évitées par la suite.

Enfin le principe sed perseverare diabolicum qui signifie clairement que, si on a le droit à l'erreur, la répétition des mêmes erreurs est évidemment fatale. 
Après cette phase d'acquisition des prérequis vient l'apprentissage d'un savoir et d'un savoir-faire translationnel. Les textes sont abordés par étapes, dont la succession constitue l'ensemble de l'opération traduisante. Ces étapes sont les suivantes: premier contact avec le texte, méthodes et objectifs de la lecture exploratoire, collecte des documents nécessaires à la traduction, dépouillement thématique et terminologique des documents en fonction de la traduction, saisie d'une terminologie ponctuelle (dictionnaire du texte) en langue source à l'aide d'un logiciel de terminologie, appariement terminologique langue source-langue cible en fonction de la hiérarchie des sources (degré de fiabilité) et de l'obligation de traçabilité lexicale (indication précise des sources), problèmes et solutions pour les correspondants introuvables, validation de la liste terminologique par le donneur d'ordre ou, à défaut, par l'enseignant, saisie du premier jet à l'aide d'un logiciel de traitement de texte en respectant ses qualités indispensables vérifiées par autorévision et dictées par l'obligation de résultat qui rend la révision intellectuellement et économiquement possible (respect de la présentation ${ }^{5}$, justesse de l'orthographe, complétude, permanence lexicale et stylistique, impératif de cohérence et de logique, exactitude des données chiffrées, marquage à l'intention du réviseur des termes et passages dont le traducteur n'est pas sûr), révision en tant que valeur ajoutée de l'assurance qualité fondée sur le principe de la division entre production et contrôle (Chaballe et Klein 1991), finalisation du «traduit» en tant que produit destiné à un client avec éventuellement une traduction page à page réalisée en publication assistée par ordinateur (PAO).

Une telle pédagogie suppose des matériels et outils informatiques adéquats, allant du traitement de texte au logiciel de traduction assistée par ordinateur (TAO) (surtout utile pour les mises à jour et les stéréotypes) en passant par le logiciel de terminologie (de préférence intégré à la TAO), mais aussi une organisation stricte dans la division du travail afin de permettre la constitution de petits groupes efficaces et responsables vis-à-vis d'euxmêmes, des autres acteurs de l'opération traduisante (documentalistes, terminologues, traducteurs et réviseurs) et last but not least du donneur d'ordre.

\section{De l'exercice d'école à la commande d'un donneur d'ordre}

Au début de la formation des traducteurs, les textes proposés aux étudiants sont des exercices d'école, c'est-à-dire des textes dont la traduction, quelle qu'en soit la forme ${ }^{6}$, n'a pas de véritable destinataire. Au fur et à mesure de la formation, il est souhaitable de présenter aux candidats de véritables traductions demandées par de véritables donneurs d'ordres. Pour ce faire, plusieurs solutions sont possibles, mais peu sont praticables en Belgique francophone: pas ou peu de junior-entreprises, pas de $1 \%$ formation de la masse salariale, pas d'obligation de stages, etc. De ce point de vue, la France est beaucoup mieux placée que nous. Il faut donc se rabattre sur d'autres possibilités qui ne sont pas moins intéressantes.

Nous en pratiquons deux au cours de la quatrième année d'études.

La première consiste à lancer un appel d'offres aux collègues des autres facultés (sciences, sciences appliquées c'est-à-dire les écoles d'ingénieurs, médecine, sciences économiques, sciences psycho-pédagogiques...) qui ont souvent des textes ou des ouvrages dans une langue étrangère qu'ils ne comprennent pas et dont ils souhaitent prendre connaissance pour leurs recherches ou leurs enseignements. Cela constitue un véritable «vivier de textes» débouchant sur une traduction en situation réelle. En fait, il s'agit d'un échange de bons procédés. La traduction est dirigée conjointement par le spécialiste de la matière et par le professeur de traduction.

Le spécialiste de la matière fait un exposé introductif sur le sujet, indique les ouvrages de référence utiles, se tient à la disposition pour répondre aux questions, valide la liste terminologique en langue cible, procède à une relecture intégrale - éventuellement en présence des étudiants concernés - afin d'en valider la qualité scientifique. 
Le professeur de traduction organise le travail, y compris le calendrier, contrôle le dépouillement thématique et terminologique, vérifie la justesse de la liste terminologique en langue source, effectue une première validation de la liste terminologique appariée, suit l'élaboration de la traduction, fractionnée ou non, fait office de réviseur, discute les problèmes épineux avec son collègue spécialiste de la matière, fait réaliser au besoin une présentation page à page en PAO (manuels d'utilisation, feuilles d'expériences...).

Cette collaboration interdisciplinaire est particulièrement fructueuse pour toutes les parties, que ce soit au niveau de la classe ou à titre individuel lors de la confection de mémoires de fin d'études. De nombreux étudiants apprécient de ne pas «travailler pour rien», d'être guidés par un spécialiste de la matière et, aussi, de voir leur produit finalisé parfois inclus, du moins en partie, dans le cursus d'une autre faculté.

Quant au service demandeur, il dispose de l'information traduite à laquelle il n'aurait probablement pas eu accès faute de moyens financiers.

La seconde possibilité fait appel à un traducteur chevronné, connu pour la qualité de son travail, compétent pour les besoins documentaires, au fait des innovations techniques appliquées au poste de travail du traducteur (Chaballe et Klein 1991) et à la pointe en matière de publications, ouvrages de référence, dictionnaires, encyclopédies... Il intervient en qualité de professeur visiteur et dirige, une fois par mois, pendant une demi-journée, un atelier de traduction. La présence et l'enseignement d'un professionnel à temps plein complète et enrichit la formation habituelle. Ce contact avec un traducteur grandeur nature permet d'aborder, au delà des traductions réalisées à partir de textes réellement destinés à la traduction, des questions spécifiques à la vie professionnelle quotidienne; au statut du traducteur et à l'évolution du marché.

\section{CONCLUSION}

Les exigences croissantes que requiert la profession de traducteur ont contraint les enseignants à adapter la formation. Jusqu'à présent, cette adaptation a dû se dérouler dans le cadre - étriqué - des programmes existants, ce qui équivaut à gérer de la façon la plus intelligente possible un état de pénurie permanente. En effet, les programmes officiels des cours dispensés dans les instituts supérieurs de traducteurs et d'interprètes datent du début des années soixante, époque où la micro-informatique n'existait pas, où la terminologie faisait ses premiers pas et où il était fréquent de rendre des traductions sous forme manuscrite. Depuis, les changements ont été importants, la profession s'est affirmée, elle s'est développée et a évolué, mais les programmes de formation, eux, sont restés, malgré leur caractère partiellement obsolète et, surtout, leurs lacunes de plus en plus criantes. De tous les côtés, des voix s'élèvent pour réclamer une refonte des programmes, mais, comme il semble plus facile de déplacer les montagnes, les enseignants de la traduction sont obligés - en attendant une hypothétique réforme «encommissionnée ${ }^{7}$ » depuis de nombreuses années déjà - de faire preuve d'imagination, de trouver des palliatifs, de «tricher» avec les intitulés existants, sous peine de former d'emblée des traducteurs «vieux jeu».

Heureusement, la plupart des professeurs de traduction y sont préparés. Ils savent, en fin de compte, que la profession de traducteur est un métier de mutant, car elle exige de pouvoir s'adapter à des sujets, des textes, des circonstances, des objectifs, des clients... différents et de pouvoir évoluer avec eux. En d'autres termes, ils usent très largement d'une liberté académique qui leur est dictée par leur conscience professionnelle, leur connaissance du monde de la traduction et le respect intellectuel et pédagogique qu'ils doivent à leurs étudiants. S'ils agissaient autrement, les jeunes traducteurs diplômés ne le leur pardonneraient pas, car ils éprouveraient, à juste titre, le sentiment d'avoir été spoliés. 
Notes

1. «Mitteilungsblatt für Dolmetscher und Übersetzer (MDÜ)», Vergabe von Übersetzungsaufträgen an freiberufliche Übersetzer seitens der Europäischen Gemeinschaften, Heft 1/1993, S. 16-17.

2. GERIN, Sabine (1990): Vous avez dit traducteur? Enquête sur le traducteur en Belgique, mémoire de fin d'études, Université de Mons-Hainaut, École d'Interprètes Internationaux, 1990, p. 51.

3. Ibid., p. 64

4. Ibid., p. 84.

5. Les règles que nous appliquons pour la présentation sont celles du Lexique des règles typographiques en usage à l'imprimerie nationale et du Code typographique.

6. GOUADEC, Daniel, (1989) p. 22 et suiv.

7. Belgicisme signifiant qu'un problème est renvoyé aux calendes grecques, parce qu'il est confié à une commission chargée de l'examiner.

\section{RÉFÉRENCES}

CHABALLE, Louis Yvon et Jean KLEIN (1992) : «La station de travail du traducteur professionnel et ses implications pédagogiques», L'environnement traductionnel. La station de travail du traducteur de l'an 2001, Actes du Colloque de Mons, Presses de l'Université du Québec, AUPELF-UREF.

CHABALLE, Louis Yvon et Jean KLEIN (1991) : «La révision de la traduction et son apprentissage», Sprache und internationale Wirtschaftsbeziehungen, Praetorius Limited, Nottingham.

Code typographique, Choix de règles à l'usage des auteurs et professionnels du livre, 1989, seizième édition, Fédération C.G.C. de la Communication, Paris.

DURIEUX, Christine (1988): Fondement didactique de la traduction technique, coll. Traductologie, $\mathrm{n}^{\circ} 3$, Didier Érudition, Paris.

GERIN, Sabine (1990): Vous avez dit traducteur? Enquête sur le traducteur en Belgique, mémoire de fin d'études, Université de Mons-Hainaut, École d'Interprètes Internationaux.

GOUADEC, Daniel (1989): Le traducteur, la traduction et l'entreprise, afnor gestion, Paris.

GOUADEC, Daniel (1990): Terminologie, constitution des données, afnor gestion, Paris.

KLEIN, Jean (1987): «Die Rolle des Konsumenten bei der Übersetzung von Gebrauchsanweisungen : dargestellt am Beispiel der Übersetzung aus dem Deutschen ins Französische», Interface, Tijdschrift voor toegepaste linguistiek, n_ 1.2, Bruxelles.

Lexique des règles typographiques en usage à l' Imprimerie nationale, 1990, Imprimerie nationale, Paris. 